\title{
The Construction of the Major Clusters in Higher Vocational Colleges from the Perspective of Industrial Cluster
}

\author{
Qingtang ZHANG, \\ Jiangyin Polytechnic College, \\ Jiangyin 214405, Jiangsu, China
}

\author{
Chengying SHEN \\ Jiangyin Polytechnic College, \\ Jiangyin 214405, Jiangsu, China
}

\begin{abstract}
The development of industrial cluster plays a significant influence on the major construction of higher vocational colleges. In this paper, in combination with the practice of Jiangyin Polytechnic College, the interaction relationship between the construction of the major clusters in higher vocational colleges and the development of the regional industry clusters is analyzed, and then the significance and idea of constructing the major clusters are introduced from the perspective of industrial cluster.
\end{abstract}

Keywords: Industrial Cluster; Higher Vocational Colleges; the Construction of Major Clusters

\section{Introduction}

The Decision of the State Council of China about Accelerating the Development of the Modern Vocational Education System, promulgated in May 2014, clearly shows that it is necessary to scientifically and rationally set up majors, improve the dynamic adjustment mechanism of majors along with the development of industries and emphatically promote the ability to train the talented personnel oriented at the fields such as the modern agriculture, advanced manufacturing, modern service, strategic emerging industries and social management, and the construction of ecological civilization. This clearly indicates a direction for the construction of the majors in higher vocational colleges. Higher vocational colleges can play an important role, only if they make a comprehensive analysis of the characteristics of the regional industry clusters, formulate the right personnel training goals, give great impetus to the construction of major clusters, train and transport high-quality technical personnel as many as possible, and constantly create talent bonuses.

\section{Analysis on the adaptability between major cluster and industrial cluster}

\subsection{The concept of industry cluster}

In 1990, Professor Michael Porter in Harvard published his typical works the Competitive Advantage of Nations. Industrial cluster is a group composed of interactively correlated companies, suppliers, financial institutions, industries, and specialized associations in a specific field in a particular place [1].

\subsection{The development of industrial cluster}

\subsubsection{The situation of foreign industrial clusters}

During the general recession of the European countries, there were a large number of small and medium-sized enterprises in the agricultural regions of the North-East and middle Italy, but they were difficult to achieve a great development because of their limited size and strength, although they fiercely competed with each other and tried hard to manage business. In the process of exploring a way for future developments, these small and medium-sized enterprises have begun to carry out cooperation and exchange by relying on the intensive advantages. Then, they have more and more frequently connected with each other in the 
process of production and operation, and have gradually formed a highly flexible specialized production collaboration network. The cluster model promotes the small and medium-sized enterprises to develop an effective competition and cooperation relationship between each other, and they have united each other to develop shoulder by shoulder. Thus, they have won a regional advantage and built up a regional brand. 2.2.2 The development of the domestic industry cluster

After the reform and opening-up policy was implemented in China, the industrial cluster development model of developed countries has been used as reference, and industrial clusters have successively emerged in some regions and industries. For example, industrial clusters featured by "one village, one brand" and "one town, one industry" have appeared in Jiangsu, Zhejiang, Guangdong and other coastal areas, and many of them have developed into the high-quality industrial clusters with large production scales, high production efficiency, complete industrial chains, and high social reputation, and have become the main forces of driving the local economic growth and rural industrialization [2].

\subsection{The interaction between industrial cluster and major cluster}

The development of industry cluster is not only an important basis for higher vocational colleges to construct major clusters, but also an important power to promote the construction of major clusters in higher vocational colleges. Moreover, enterprise equipment and production sites provide important material conditions for the construction of major clusters.

Jiangyin Polytechnic College is taken as an example. The school is located in Jiangyin, Jiangsu province. Jiangyin is the core area of Suzhou-Wuxi-Changzhou and one of the most developed counties in China, and its economic aggregate and comprehensive competitiveness are in the list of China's top counties/cities. There have been more than 30,000 enterprises in Jiangyi, in which 155 are group enterprises, 14 are in the list of China's top 500 manufacturing enterprises, 10 are in the list of China's top 500 enterprises, 12 are in the list of China's top 500 private enterprises, and 36 are listed companies (HEILAN, SUNSHINE, and FASTEN). Thus, the well-known "Jiangyin phenomenon" and "Jiangyin plate" have emerged. Jiangyin adheres to the "two-wheel driving model" of high-end manufacturing and modern service, so that 8 pillar industries (electronic information, machinery equipment, automobile and parts, special metallurgy, new materials, textile and garment, biomedicine, and energy conservation and environmental protection). Jiangyin Polytechnic College, cored at special brand majors and closely combining with the actual conditions of regional economic and social development, has decisively integrated 35 majors into textile and apparent major cluster, creative design major cluster, mechanical manufacturing and automation technology major cluster, and modern business service major cluster, and the major clusters meeting the development demands of the regional industries. These major clusters have well served Jiangyin and the development of the industrial clusters in the surrounding areas, and have been consistently praised by all walks of life.

\section{The practical significance of the construction of major clusters from the perspective of industrial cluster}

The Modern Vocational Education System Construction Plan (2014-2020) printed by the Sixth Commission of the Ministry of Education clearly shows that it is necessary to promote the vocational education to melt into the entire economic and social development and reform and opening-up process, and establish a connection between major setup and industrial requirements, between curriculum contents and professional standards, and between teaching process and production process, so as to make 
higher vocational education adaptive to technical progress, production mode change and social public services, and boost economic quality and efficiency upgrades. The statement promotes the height of the major cluster construction up to the national level.

\subsection{The construction of major cluster is a demand of optimizing the structure of major structure}

The construction of major cluster is one of the most important aspects of the intension construction in higher vocational colleges, but also a concentrated reflection to the school education quality and level. The construction means higher vocational colleges have shifted the development ideas and ways to the "intensive education" pursuing quality, benefits and scales and the intension-oriented development paths, after a period of time of "extensive education" [3].

\subsection{The construction of major cluster is a demand of reducing the management difficulties}

In general, a lot of work needs to be starting from scratch if a new major is set up, including hiring teachers, establishing curriculum system, organizing teaching materials, and planning and constructing training sites. Therefore, the workload is self-evident. With the construction of major construction, higher vocational colleges can avoid the above contradictions by relying on the professional manpower and material resources of other majors of a cluster, so that the complexity and cost of the construction of major cluster are greatly reduced.

\subsection{The construction of major cluster is a demand of deepening the integration of production with education}

Major cluster is beneficial to expand and deepen the cooperation between colleges and enterprises. It can not only transport all aspects of talents for enterprises, and also can help individual students learn multi-aspect knowledge and skills with the aid of school-enterprise cooperation opportunity. Major cluster adapts to the characteristics of the regional industry cluster [4].

\subsection{The construction of major cluster is a demand of building up brand characteristics}

Higher vocational colleges, in the process of pursuing special development and creating differentiated competitive advantages, strengthen the self-intension construction using major cluster, in order to improve the quality of personnel training and the core competitiveness. This is an internal driving force for higher vocational education's self-development. Major cluster is often a highlight of the higher vocational colleges to admit new students, but also a focal point of the employing units in talents recruitment. Its advantages play throughout the whole of admissions and employment.

\section{The idea of the major cluster construction from the perspective of industrial cluster}

The construction of major cluster from the perspective of industrial cluster must be started from the concept of service and cored at brand or special majors. Also, major cluster must be scientifically planed and constructed around industrial chain, professional post group, and discipline foundation, so as to train the distinct majors that can meet the great demands of enterprise, serve the society powerfully, provide good employment prospect for students, and receive good comments from all walks of life. Thus, a connection is built between major setup and industry demand, between curriculum contents and professional standards, between teaching process and production process, between graduation certificate and professional qualification, and between vocational education and lifelong learning [5]. 


\subsection{Building a scientific and reasonable major cluster curriculum system}

Indistinctive major characteristics and unreasonable courses are important problems in the traditional personnel training model. Under the new situation, an important aspect in the construction of the curriculum system for major cluster is to clearly define the cross-relationship between major and courses. Courses must include theoretical courses and practical courses, and they are glues between different majors to construct a cluster. Therefore, it is necessary to know well the common and different courses between majors. Next, the curriculum construction must be specially focused.

\subsection{Giving full play to the characteristics of higher vocational colleges and regions}

Different from the practice of general undergraduate colleges and universities to set up courses based on the discipline theory system, higher vocational colleges often set up majors based on the professional requirements and the market. The construction law of major cluster lies in actively adapting to the constantly changing adjustments of the social industries, industry structure, and professional posts. In an objective sense, major cluster requires higher vocational colleges to meet the condition and construct several major clusters with the same or closely related technical foundations and the same requirements for major technical courses and basic technical abilities to cover a technical or service field [6].

\subsection{Dynamically controlling the construction scale of major cluster}

To control of cluster scale, the correlation and complementary functions between different majors must be clearly defined, namely, which majors to be clustered and which major to be used as dominant. Based on correlation, the most important, well-improved, and demonstrative brand major can be used as a core, and other majors can be used as auxiliary specialties.
Nevertheless, each major must be aware of being a master in the cluster and give full play to the subjective initiative, so as to make every effort to the construction of the cluster.

\subsection{Building up an excellent major faculty}

Industrial cluster is not only a collection of production and management, but it is a collection of talents in essence. To obtain an industrial development, it is necessary to set up an outlook on talented personnel and create personal introduction mechanism. Higher vocational colleges produce a large number of talents from year to year and add fresh blood for the development of industries. Talented personnel training must rely on a powerful faculty, and therefore, higher vocational colleges must build a major faculty with a reasonable structure, excellent quality, and integration of theories with practice.

\section{Conclusion}

The construction of major cluster is the most efficient form in the major construction of higher vocational education. The advantage of major cluster is embodied in the connection and interaction with the local industries. In the process, industrial cluster is not only an instruction for the idea of major cluster, but also foundation and criterion. There are great common points between industrial cluster theory and major cluster construction. In the actual operation process, the idea of industry cluster plays a practical guiding role in the construction of major cluster.

\section{Acknowledgement}

This paper aided financially by the research project of the higher education reform in Jiangsu province in 2013 (2013JSJG341). 


\section{References}

[1] Yongxin WANG. Discussion on the Application of the Industrial Cluster Theory [J]. Journal of the Small and Medium-sized Enterprise Management and Technology, 2008, 10.

[2] Chunyan LI. An Empirical Research on the Influence of Industrial Cluster Advantages on the Regional Brand- Investigation and Analysis based on the Perspective of Cluster Enterprise [D]. Master Degree Thesis of Master of Lanzhou University.

[3] Xi ZHAO. Thinking and Understanding of the Program Cluster Construction Practice in Higher Vocational Colleges [J]. Journal of Higher Vocational Education (Tianjin Vocational University), 2011. 06.
[4] Feng SUN. Study on the Program Cluster Establishments in Higher Vocational Colleges from the Perspectives of the Coordination between Program Cluster and Industrial Clusters [J]. Higher Education Research, 2014, 07.

[5] The Decision of the State Council about Accelerating the Development of the Modern Vocational Education System [J]. Journal of vocational Technology, 2014, 08.

[6] Caiyun ZHANG, Dong ZHAO, Zhongjian ZHAO. Consideration on the Coordinated Development of Program Cluster Construction and Industry Cluster [J]. Journal of Huaibei Normal University Journal (Philosophy and Social Sciences Edition), 2014, 11. 